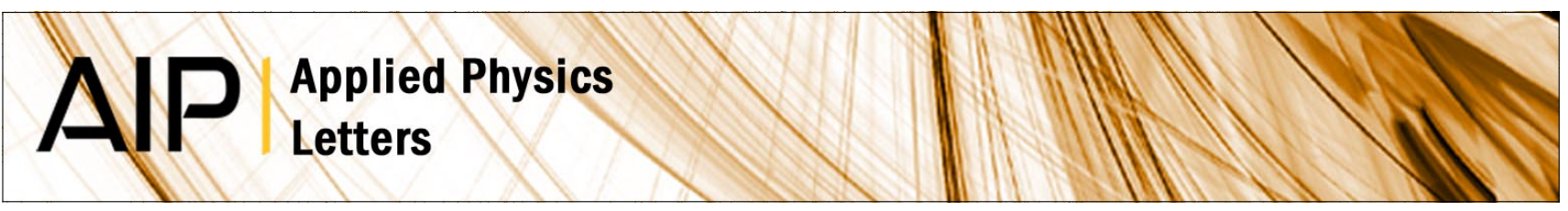

\title{
Tuning the polarization state of enhanced transmission in gratings
}

David Crouse, Alastair P. Hibbins, and Matthew J. Lockyear

Citation: Appl. Phys. Lett. 92, 191105 (2008); doi: 10.1063/1.2924317

View online: http://dx.doi.org/10.1063/1.2924317

View Table of Contents: http://apl.aip.org/resource/1/APPLAB/v92/i19

Published by the American Institute of Physics.

\section{Related Articles}

In situ etched gratings embedded in AIGaAs for efficient high power 970nm distributed feedback broad-area lasers

Appl. Phys. Lett. 100, 201115 (2012)

Taming the thermal emissivity of metals: A metamaterial approach

Appl. Phys. Lett. 100, 201109 (2012)

Growth rate and start current in Smith-Purcell free-electron lasers

Appl. Phys. Lett. 100, 191101 (2012)

Powerful surface-wave oscillators with two-dimensional periodic structures

Appl. Phys. Lett. 100, 143510 (2012)

Direction switching and beam steering of cholesteric liquid crystal gratings

Appl. Phys. Lett. 100, 131909 (2012)

\section{Additional information on Appl. Phys. Lett.}

Journal Homepage: http://apl.aip.org/

Journal Information: http://apl.aip.org/about/about_the_journal

Top downloads: http://apl.aip.org/features/most_downloaded

Information for Authors: http://apl.aip.org/authors

\section{ADVERTISEMENT}
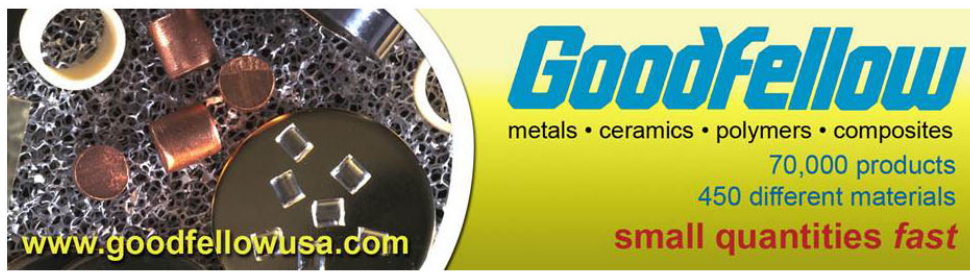


\title{
Tuning the polarization state of enhanced transmission in gratings
}

\author{
David Crouse, ${ }^{1, a)}$ Alastair P. Hibbins, ${ }^{2}$ and Matthew J. Lockyear ${ }^{2}$ \\ ${ }^{1}$ Department of Electrical Engineering, The City College of New York, Steinman Hall, Room 614, \\ 140th St. at Convent Ave., New York, New York 10031, USA \\ ${ }^{2}$ Electromagnetic Materials Research Group, School of Physics, University of Exeter, Stocker Road, \\ Exeter EX4 4QL, United Kingdom
}

(Received 25 March 2008; accepted 21 April 2008; published online 14 May 2008)

\begin{abstract}
The polarization characteristics of enhanced transmission of lamellar gratings with structural dimensions on the subwavelength scale were studied and experimental results were compared to numerical models. The ability to tune the polarization state of the transmitted beam by varying the grating's structural parameters is discussed. Gratings were fabricated and tested in the microwave spectral region, and the results were compared to theoretically modeled results. Enhanced transmission produced by cavity modes was experimentally verified for both $s$-polarized and $p$-polarized incident beams of light. Applications of these results to photonic devices in the visible, infrared, and microwave spectral regions are discussed. (C) 2008 American Institute of Physics.
\end{abstract}

[DOI: $10.1063 / 1.2924317]$

There has been much interest in the phenomenon of enhanced transmission in periodically patterned metal structures, both in two-dimensionally periodic hole arrays and in one-dimensionally (1D) periodic transmission grating structures. ${ }^{1-14}$ Enhanced transmission is a phenomenon in which light, incident on a periodically patterned optically thick metal structure, is transmitted with a transmittance $(T)$ substantially greater than the ratio of the area of the grooves $\left(A_{\text {groove }}\right)$ to the total area of the structure on which the light is incident $\left(A_{\text {total }}\right)$, i.e., $T>A_{\text {groove }} / A_{\text {total }}$. This phenomenon was first attributed to surface plasmons that are oriented parallel to the surface [denoted as horizontally oriented surface plasmons (HSPs)]. ${ }^{1,2}$ However, if enhanced transmission only occurred via HSPs, then in 1D gratings, only $p$-polarized incident light would exhibit this phenomenon and not $s$-polarized light. ${ }^{11}$ This result would greatly limit the use of enhanced transmission in the development of many practical optoelectronic devices. Other characteristics of HSPs that are often undesirable for the development of optoelectronic devices are the small bandwidth of their transmission peaks, high absorption (relative to other optical modes), and the position of the amplified electromagnetic field intensities associated with HSPs. ${ }^{9,11}$ In this work, the experimental verification of enhanced transmission produced by $p$-polarized and $s$-polarized cavity modes (CMs) in lamellar gratings is theoretically and experimentally demonstrated.

It has been experimentally and theoretically shown that $\mathrm{CMs}$ in lamellar gratings produce enhancements in transmission for $p$-polarized incident light and that their properties (e.g., bandwidth, electromagnetic field profiles) and dependencies on wavelength, angle of incidence, and structural geometries are significantly different from HSP-induced transmission. ${ }^{7-9,11}$ In lamellar gratings with period $\Lambda$ and with grooves with a height of $h$, a width of $c$, and the dielectric material filling the groove with a dielectric constant $\varepsilon_{\text {groove }}$, CMs are produced within the grooves by waveguide modes for $s$-polarized light and either waveguide modes or vertically oriented surface plasmons (VSPs) on the walls of

${ }^{a)}$ Electronic mail: dcrouse@ccny.cuny.edu. the grooves for $p$-polarized light. ${ }^{11}$ As discussed in Ref. 11 these modes have a high degree of wavelength, bandwidth, and polarization tunability and can, with the use of low loss metals and dielectrics, transmit close to $100 \%$ of incidence light. ${ }^{11}$ The $p$-polarized CMs have strong dependencies on $h$ and $\varepsilon_{\text {groove }}$ and a weak dependence on $c$. Also, $p$-polarized CMs can have a strong dependence on $\Lambda$, especially when $\Lambda$ is such to produce a Wood-Rayleigh (WR) anomaly or a HSP at a wavelength close in value to that of the CM. The $s$-polarized CMs have strong dependencies on $h, c$, and $\varepsilon_{\text {groove }}$ and a weak dependence on $\Lambda$. The following series of steps (in order) can be used to tune and align at a single wavelength the enhanced transmission peaks associated with both an $s$-polarized CM and a $p$-polarized CM for a simple lamellar grating.

(1) Choose a grating period $\Lambda$ so that the onset of first order diffraction (for both the substrate and superstrate) is at a smaller wavelength than the wavelength at which enhanced transmission is desired.

(2) Choose an initial value for $c, h$, and $\varepsilon_{\text {groove }}$ to get the $s$-polarized and $p$-polarized CMs in the approximate wavelength range desired. The larger $h$ is, the closer spaced (in wavelength) the CMs are for each polarization. The larger the aspect ratio $h / c$ is, the higher the $Q$-factor of the CMs. Too large of an aspect ratio, however, will produce large absorption for real metals. Importantly, grooves that are wide enough to support a $s$-polarized CM will generally allow $p$-polarized light to be transmitted in appreciable amounts even when a $p$-polarized CM is not excited. One way around this problem is to use a high-index dielectric that does two things: (1) increases the effective optical width and height of the groove by a factor of $\sqrt{\varepsilon_{\text {groove }}}$ and (2) increases the optical impedance mismatch, thereby reducing the $p$-polarization transmission when a $p$-polarized $\mathrm{CM}$ is not excited. The term optical impedance mismatch is used in this context as it is used to describe reflection and transmission of light incident on simple freestanding dielectric films; the Fresnel equations pre- 


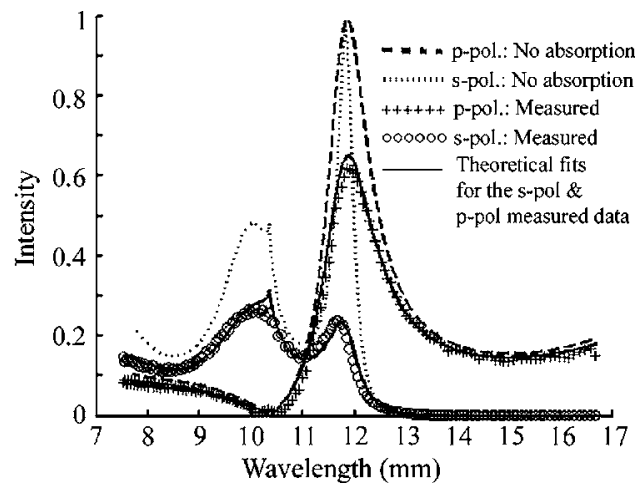

FIG. 1. The numerically modeled (both with and without absorption in the elastomer filling the grooves) and experimental data of $p$-polarization and $s$-polarization, normal incidence transmittance for a lamellar grating with Al contacts $\left(\varepsilon_{\mathrm{Al}}=-10^{4}+i 10^{7}\right)$, a pitch of $10.343 \mathrm{~mm}$, groove width of $3.821 \mathrm{~mm}$, thickness of $6.045 \mathrm{~mm}$, and groove dielectric of 2.8 . A $p$-polarized $\mathrm{CM}$ mode is aligned with an $s$-polarized $\mathrm{CM}$ mode at $\sim 25.188 \mathrm{GHz}(\lambda=11.9 \mathrm{~mm})$ with both having close to $100 \%$ transmittance. There is a good fit between the experimental and numerically modeled fitted curves that include absorption in the elastomer.

dict that, in the absence of resonance modes and other complicating factors, transmission of light through a film decreases as the difference between the dielectric constants of the air and thin film increases.

(3) Vary the groove height $h$ to get the $p$-polarized $\mathrm{CM}$ at the desired wavelength.

(4) Vary the groove width $c$ to align the s-polarized CM with the $p$-polarized CM.

The structure that was fabricated in this work is a simple lamellar grating that is designed to transmit both $s$-polarized and $p$-polarized microwaves at a frequency of $25.188 \mathrm{GHz}$ $(\lambda=11.91 \mathrm{~mm})$. The grating design, both numerically modeled and tested, has aluminum contacts $\left(\varepsilon_{\mathrm{Al}}=-10^{4}+i 10^{7}\right)$, $\Lambda=10.3428 \mathrm{~mm}, c=3.8211 \mathrm{~mm}, h=6.045 \mathrm{~mm}, \varepsilon_{\text {groove }}=2.8$, and air for the substrate and superstrate. Note that CMs, HSPs, VSPs, WRs, diffraction, and all other optical effects occur in the microwave as they do in the infrared and visible spectral regions, but the CMs and diffraction features occur at wavelengths that scale with groove height and width and grating pitch. Shown in Figs. 1-3 are the numerically modeled and experimental transmittance (Fig. 1), the full $s$-polarization $\omega$ - $k$ transmittance profiles (Fig. 2), and the numerically modeled electric field intensities of the $25.188 \mathrm{GHz} s$-polarized CMs (Fig. 3). Due to space limitations, similar graphs for the $p$-polarization are not included

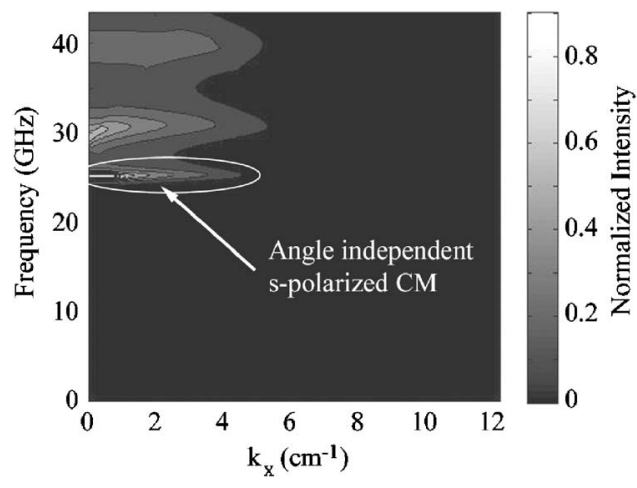

FIG. 2. The numerically modeled transmittance for an $s$-polarized beam.

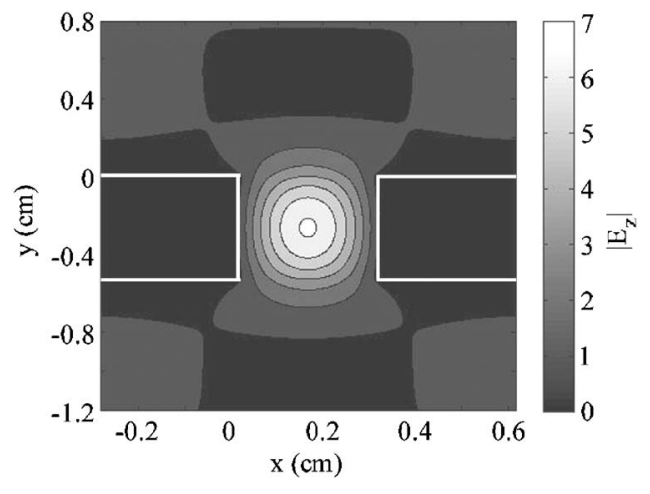

FIG. 3. The numerically modeled electric field amplitude, given a unit amplitude, normal incident $s$-polarized $25.188 \mathrm{GHz}(\lambda=11.9 \mathrm{~mm})$ microwave beam.

here, but many examples are given in Refs. 9-11. All of the properties of CMs of both polarizations, that are extensively discussed in Refs. 8, 9, and 11, can be seen in these figures, including their high transmittance and small angle of incidence dependence. The experimental sample is constructed by machining a set of identical grooves, each with the dimensions previously mentioned with the dielectric material in the grooves being an elastomer (Dow Corning ${ }^{\circledR}$ Sylgard ${ }^{\circledR}$ 184 silicone encapsulant). The real part of the permittivity of the elastomer has been determined in a previous work to be $\sim 2.8 \mathrm{GHz} .{ }^{12}$ Linearly polarized microwave radiation from a standard gain horn is collimated by using a spherical mirror and impinges upon the sample at normal incidence. A continuous wave source sweeps the frequency in bands of 18 $\leqslant \nu \leqslant 26.5$ and $26.5 \leqslant \nu \leqslant 40 \mathrm{GHz}$ (i.e., $7.5 \leqslant \lambda \leqslant 16.7 \mathrm{~mm}$ ) and feeds the fixed position antenna. Before striking the sample, the incident beam passes through an aperture of a broadband microwave absorbing material in order to restrict the incident beam spot to the useful sample area. Furthermore, in order to obtain averaging of the transmitted signal over a large number of grating periods, the transmitted beam is collected by using another spherical mirror before being focused into a second horn antenna and detector. The polarization of both the incident beam and that detected can be altered via simple rotation of each horn antennas about its central axis. The experimental transmittivity data, setting both the incident and detected polarizations to either $p$-polarization or $s$-polarization, normalized to a spectrum in the absence of the sample, are shown in Fig. $1(+$ and $\bigcirc$, respectively). As is seen in Fig. 1, the experimental transmittivities are substantially reduced relative to the predicted values obtained by numerical modeling; however, once a small absorptive component, associated with a Debye dielectric response of the polymer and impurities, is included in the dielectric constant of the elastomer used in the modeling, the modeled and experimental curves match very well. The amount of optical energy that is absorbed depends on the field intensity of the CM integrated over the volume of the cavity. For the two $25.188 \mathrm{GHz}$ CMs used in this work, the $s$-polarized CM has a field intensity that is amplified by a factor of $\sim 50$ (relative to the incident beam) and is spread over a large volume within the groove, the $p$-polarized CM has a field intensity of only 25 and is spread over a smaller volume within the groove. Therefore, for the two CMs used in this work, more absorption is expected when an $s$-polarized $\mathrm{CM}$ is excited than when a $p$-polarized $\mathrm{CM}$ is 
excited. By fitting the experimental data to the modeling, it was found that the structure that was fabricated had a groove width of $3.824 \mathrm{~mm}$ and a dielectric constant for the groove of $\varepsilon_{\text {groove }}=2.75+i 0.0945$. The magnitude of these dielectric losses can be reduced by the use of crystalline powders or other low loss materials instead of the silicone encapsulant. ${ }^{13}$

The abilities to control the polarization state of the transmitted beam and the directional flow of light can be applied to numerous device applications including broadband polarizers, photodetectors, and polarimetric sensors. Even though the simple single-groove-per-period lamellar gratings can simultaneously perform several functions (e.g., polarizing, wavelength filtering, channeling, and localizing light), further increases in functionality and performance can be obtained by studying multiple-groove-per-period compound gratings. ${ }^{14}$ In conclusion, a procedure to control the polarization state of the transmitted beam has been described. A simple lamellar grating structure that exhibits enhanced transmission for both $p$-polarized and $s$-polarized incident beams of light at the same wavelength was fabricated and characterized.
The Cornell Center for Nanoscale Systems in Information Technologies and the National Science Foundation under Grant No. ECC-0646547 supported the work performed by D.C. and A.H. and M.L. were supported by the EPSRC. The authors also wish to thank Dr. Thomas James for helpful discussions and other contributions to this work.

${ }^{1}$ T. W. Ebbesen, H. J. Lezec, H. F. Ghaemi, T. Thio, and P. A. Wolff, Nature (London) 391, 667 (1998).

${ }^{2}$ D. E. Grupp, H. J. Lezec, T. Thio, and T. W. Ebbesen, Adv. Mater. (Weinheim, Ger.) 11, 860 (1999).

${ }^{3}$ R. W. Wood, Philos. Mag. 4, 396 (1902).

${ }^{4}$ A. Hessel and A. A. Oliner, Appl. Opt. 4, 1275 (1965).

${ }^{5}$ U. Fano, J. Opt. Soc. Am. 31, 213 (1941).

${ }^{6}$ M. M. J. Treacy, Phys. Rev. B 66, 195105 (2002).

${ }^{7}$ A. Barbara, P. Quemerais, E. Bustarret, and T. Lopez-Rios, Phys. Rev. B 66, 161403(R) (2002).

${ }^{8}$ D. Crouse and P. Keshavareddy, Opt. Express 13, 7760 (2005).

${ }^{9}$ D. Crouse, IEEE Trans. Electron Devices 52, 2365 (2005).

${ }^{10}$ D. Crouse and P. Keshavareddy, J. Opt. A, Pure Appl. Opt. 8, 175 (2006).

${ }^{11}$ D. Crouse and P. Keshavareddy, Opt. Express 15, 1415 (2007).

${ }^{12}$ F. Yang and J. R. Sambles, J. Phys. D: Appl. Phys. 35, 3049 (2002).

${ }^{13}$ Y. Imanaka, M. Takenouchi, and J. Akedo, J. Cryst. Growth 275, e1313 (2005).

${ }^{14}$ D. Crouse, E. Jaquay, A. Maikal, and A. P. Hibbins, Phys. Rev. B (in press). 